\title{
Clinical Trial Highlights - GLP-1 agonists
}

\author{
Kevin McFarthing $P h D^{*}$, Danielle Larson $M D^{* *}$ and Tanya Simuni $M D^{* *}$
}

\author{
* Parkinson's advocate, Oxford, UK \\ ** Parkinson's Disease and Movement Disorders Center, Northwestern University Feinberg School of Medicine, \\ Chicago, IL, USA \\ $\S$ To whom correspondence should be addressed at kevinDOTmcfarthingATinnovationfixerDOTcom
}

\section{Introduction}

Parkinson's Disease (PD) is a heterogeneous, long-term neurodegenerative condition, for which there are no therapies proven to slow or stop progression. At the same time, developing a new drug from target discovery to pharmacy shelf is a long, hard and expensive journey. For that reason, almost 50 development programs are now focused on repurposing - taking drugs proven to work for one indication and investigating them for potential use in $\mathrm{PD}[1]$.

Perhaps the leading class of existing drugs currently being assessed for disease modifying benefit in PD are glucagon-like peptide 1 (GLP-1) agonists. These drugs are used in the treatment of type 2 diabetes mellitus (T2DM) where they stimulate glucose-induced insulin secretion and biosynthesis, slow down gut emptying, and inhibit glucagon secretion, thereby mediating glucose homeostasis [2].

GLP-1 agonists are based on a natural analogue of GLP-1, exendin-4, found in the saliva of the gila monster. The class has been further expanded with the development of molecules with a better pharmacokinetic profile, much longer half-life than GLP-1 itself, and very wide clinical utility.

The background and application of GLP-1 agonists to PD has been reviewed well by Athuada and Foltynie [2]. Extensive in vitro and animal model studies have identified a wide range of cellular processes influenced by the GLP-1 receptor (GLP-1R) that are of relevance to neuroprotection. There are two main pathways through which these effects occur; (i) the mitogen-associated protein kinase/extracellular signal-regulated kinase (MAPK/ ERK; also known as Ras-Raf-MEK-ERK); and (ii) PI3K/protein kinase B (AKT) [3].

GLP-1 agonists exert their neuroprotective effects in several different ways. Activation of the GLP-1R can reduce inflammation by preventing cytotoxic microglial responses and suppressing production of proinflammatory cytokines. The underlying mechanism is unclear but may be mediated downstream of the AKT pathway through nuclear factor (NF) $\kappa B$ [2]. Activation of MAPK has effects on long term potentiation and synaptic plasticity as well as stabilisation of dendritic spines [5].

GLP-1R activation has also demonstrated multiple beneficial actions on mitochondria across a range of experimental models, resulting in reduced apoptosis and oxidative stress. It can prevent the aggregation of $\beta$-amyloid and tau proteins as well as $\alpha$-synuclein. It may also indirectly influence neurogenesis. GLP-1R activation can mediate axonal growth via trophic factors such as Brain-Derived Neurotrophic Factor (BDNF) or possibly directly [2].

GLP-1 agonists have been shown to have beneficial effects in several models of PD, including 6-hydroxy dopamine (6-OHDA), both in vitro and in rats; rat lipopolysaccharide (LPS); and 1-methyl-4-phenyl-1,2,3,6tetrahydropyridine (MPTP) in mice [2]. 
There are links between T2DM and PD with epidemiological studies showing an increased risk of developing PD in patients with T2DM [4]. There is also increased insulin resistance in PD, with common pathway defects in both conditions [6]. It is therefore no surprise that drugs originally developed for T2DM are being tested to show potential therapeutic benefits in PD.

The mounting evidence of the potential utility of GLP-1 agonists in PD led to the evaluation of exenatide, a synthetic version of exendin-4, in clinical trials. First, a single-blind study of 45 patients with moderately advanced PD randomized to exenatide or control group showed improvement in UPDRS part 3 scores for those who received exenatide, with a mean improvement by 2.7 points versus a decline of 2.2 points in the control group. In addition, there was a improvement in the Mattis dementia rating scale with a mean improvement of 2.8 points in the exenatide treated patients, while the control group deteriorated by 3.5 points [7]. A follow up study of the trial cohorts showed that the improvements persisted 12 months after cessation of exenatide [8].

The next step was a double-blind, placebo-controlled Phase 2 study conducted in 60 patients with moderately advanced PD. The study confirmed the motor benefits observed in the previous study with an adjusted mean difference of -3.5 points between exenatide and placebo in MDS-UPDRS motor scores assessed medications OFF [9]. These effects were maintained but reduced at 12 weeks following the end of exenatide treatment. The cognitive benefit of exenatide seen in the open label study was not reproduced in this study. A further post hoc analysis of a wide range of non-motor symptoms showed a clinically significant improvement in the exenatide group when compared to placebo [10]. There remains a question of whether this observed benefit is driven by a symptomatic effect or by a true disease modifying effect of exenatide. This question warrants further investigation in sufficiently powered Phase 3 studies targeting newly diagnosed PD patients.

The exenatide clinical studies have set the scene well for the strong interest in the potential of GLP-1 agonists as treatments that may slow down the progression of PD. In the next section we will provide an overview of trials of GLP-1 agonists currently under way.

\section{References}

[1] McFarthing K (2020) The Parkinson's Hope List bit.ly/ParkinsonsHopeList

[2] Athauda D, Foltynie T (2016) The glucagon-like peptide 1 (GLP-1) receptor as a therapeutic target in Parkinson's disease: mechanisms of action. Drug Discovery Today 21, 802-818.

[3] Baggio LL, Drucker DJ (2007) Biology of incretins: GLP-1 and GIP. Gastroenterology, 132 (2007), 21312157.

[4] DePablo-Fernandez E et al (2018) Association between diabetes and subsequent Parkinson disease: A record-linkage cohort study. Neurology 91(2), e139-e142.

[5] Athuada D et al (2017) Is Exenatide a treatment for Parkinson's Disease? J Parkinsons Dis 7, 451458.

[6] Fiory F et al (2019) The Relevance of Insulin Action in the Dopaminergic System. Front Neurosci 13: 868.

[7] Aviles-Olmos I et al (2013) Exenatide and the treatment of patients with Parkinson's disease. J Clin Invest 123(6) 2730-6.

[8] Aviles-Olmos I et al (2014) Motor and cognitive advantages persist 12 months after exenatide exposure in Parkinson's Disease. J Parkinsons Dis 4(3) 337-344.

[9] Athuada D et al (2017) Exenatide once weekly versus placebo in Parkinson's Disease: a randomised, double-blind placebo-controlled trial. Lancet 7,390(10103), 1664-1675.

[10] Athuada D et al (2018) What effects might exenatide have on non-motor symptoms in Parkinson's Disease: a post hoc analysis. J Parkinson's Dis 8, 247-258. 


\section{GLP-1 agonists in active clinical trials}

\section{Overview}

Following the results of the aforementioned phase 2 exenatide study, there are four current trials further studying exenatide in PD: one phase 1, two phase 2, and one phase 3 study. The phase 1 single-center non-placebocontrolled study at the University of Florida is specifically examining the change in MRI brain metrics, in addition to symptomatic changes, of PD individuals after 1-year of exenatide treatment. A phase 2 study sponsored by Stockholm Health Care Services is investigating the effect of extended-release exenatide (Bydureon) injections versus placebo on progression of disease, non-motor, and motor symptoms. A second phase 2 study in South Korea is studying the effect of Peptron's novel sustained release formulation of Exenatide, PT320, on motor function. Finally, an important phase 3 study, Exenatide-PD3, is in development by the University College Hospital of London. This study intends to confirm neuroprotective effects suggested by phase 2 studies and demonstrate reproducibility across multiple centers with a large number of participants and over a longer study duration.

After exenatide paved the way for the study of GLP-1 agonists as potential neuro-protective agents in PD, there has been an escalation in the number and variety of GLP-1 agonist compounds being studied for PD. The majority of these compounds have the advantage of being repurposed FDA-approved medications with well-established safety and tolerability profiles. Additionally, these molecules have relatively robust preclinical evidence of their effects on neuro-inflammation and pathology in animal models of PD. Specifically, the Type 2 Diabetes medications lixisenatide, liraglutide, and semaglutide are being studied in phase 2 placebo-controlled trials to assess the drugs' effects on motor outcomes in PD (studied by Toulouse University Hospital with Sanofi, Cedars-Sinai Medical Center with Novo Nordisk, and Oslo University Hospital, respectively). The trials of these three re-purposed compounds all involve early-stage PD, with the primary outcome measure of change in motor function from baseline to trial end-point. A compound that is not currently FDA-approved, Neuraly's NLY01, is in a phase 2 clinical trial. This novel pegylated form of exenatide will be compared to placebo to assess its safety, tolerability, and efficacy in PD. These four phase 2 studies vary in their treatment duration; the study of semaglutide is the longest with 4 years of treatment, with 13.5 months for the study of liraglutide, 12 months for the study of lixisenatide, and 9 months for the Neuraly study of NLY01. The lixisenatide study is unique amongst these four trials in that it has a washout period of 2 months after the 12-month treatment phase. The exenatide phase 3 study has a treatment period of 96 weeks.

While this review focuses on the aforementioned compounds currently in clinical trials, there are additional molecules that are in preclinical development. This includes Kariya Pharmaceuticals' KP-405, a dual incretin receptor co-agonist, which has demonstrated efficacy in affecting neurodegeneration in animal models of PD and is moving to human studies.

\section{References}

[1] Grieco M et al. Glucagon-like peptide-1: A focus on neurodegenerative diseases. Front Neurosci Oct 2019.

[2] https://www.kariyapharma.com/

[3] http://peptron.com/ds6_1_1.html 


\section{Exenatide studies overview}

\section{Background}

There are currently four GLP-1 agonists in clinical trials for PD. The most advanced molecule in this repurposing approach is exenatide. The first trial was an open label, proof of principle study, comparing exenatide to control PD patients [1]. This was followed by a double-blind, placebo-controlled phase 2 study [2]. Both trials showed a statistically and clinically significant difference in favour of exenatide. Furthermore, follow up analyses showed that the benefits persisted after the exenatide treatment ceased [3,4]. Small sample size precluded definitive assessment of disease modifying versus symptomatic nature of the observed benefit.

There are four trials of exenatide currently in progress. The University of Florida has an investigative phase 1 study looking at how the brain and motor behaviour changes over time with exenatide. Stockholm Healthcare Services and the Karolinska Institute are aiming to correlate changes in brain metabolism, measured with FDGPET analysis, over time with observed clinical symptoms in a phase 2 study. Peptron in Korea are conducting a phase 2 investigation of their sustained-release formulation of exenatide. Finally, and most exciting from the perspective of people with Parkinson's (PwP), University College Hospital in London has started a phase 3 study of exenatide in 200 patients.

\section{References}

[1] Aviles-Olmos I et al (2013) Exenatide and the treatment of patients with Parkinson's disease. J Clin Invest 123(6) 2730-6.

[2] Athuada D et al (2017) Exenatide once weekly versus placebo in Parkinson's Disease: a randomised, double-blind placebo-controlled trial. Lancet 7,390(10103) 1664-1675.

[3] Aviles-Olmos I et al (2014) Motor and cognitive advantages persist 12 months after exenatide exposure in Parkinson's Disease. J Parkinsons Dis 4(3) 337-44.

[4] Athuada D et al (2018) What effects might exenatide have on non-motor symptoms in Parkinson's Disease: a post hoc analysis. J Parkinson's Dis 8(2) 247-258.

\section{Exenatide-PD3 - University College Hospital, London}

\section{Background}

The same research group that conducted the previous phase 2 studies are now taking exenatide into a larger phase 3 program. This will investigate efficacy over a longer time period and aims to confirm safety and tolerability in a larger patient population.

Title: Exenatide Once Weekly Over 2 Years as a Potential Disease Modifying Treatment for Parkinson's Disease (Exenatide-PD3)

\section{Phase: 3}

Status: Not yet recruiting.

Clinicaltrials.gov ID: NCT04232969

Sponsor: University College, London.

Estimated Enrolment: 200

Estimated Completion Date: September 2023. 
Study Design: A randomised, double-blind, placebo-controlled, parallel group study run at a single centre. The active drug is a $2 \mathrm{mg}$, self-administered, sub-cutaneous injection with a 2-year treatment period.

Outcome Measures: The primary outcome measure is change in the motor function as measured by MDSUPDRS part 3 in the OFF state between baseline and 96 weeks.

Secondary outcome measures are:

1. MDS-UPDRS parts 1,2 and 4 in the ON state.

2. Timed walk assessment both ON and OFF.

3. Montreal cognitive assessment (MOCA).

4. Unified dyskinesia rating scale (UDysRS).

5. Patient health questionnaire-9 (PHQ-9).

6. PD quality of life (PDQ-39).

7. Non-motor symptoms scale (NMSS).

8. Levodopa equivalent dose (LED).

9. Hauser diary (3 day) of PD state.

10. Safety and tolerability.

These assessments will also be done at baseline and 96 weeks.

\section{Comments}

The inclusion criteria are for PwP between the ages of 25 and 80 who are already on dopaminergic therapy. The Hoehn and Yahr stage must be $\leq 2.5$ in the ON state, thus excluding more advanced patients.

Given the comparatively slow degeneration rate in $\mathrm{PD}$, the treatment period of almost 2 years is a positive feature of this study. The success of the trial does, however, assume that the same rate of increase in UPDRS scores in the placebo group seen in the 48-week period in the previous studies will continue over 96 weeks in this one.

This study should also show whether the positive impact of exenatide treatment on MDS-UPDRS scores seen in prior work is a short-lived effect that dissipates with time, simply delaying a return to an expected rate of decline; or if the extended treatment may slow a further decline in disability, thus meeting the real need for people with $\mathrm{PD}$, a medicine that slows the progressive development of symptoms.

\section{Stockholm Healthcare Services/Karolinska Institute}

\section{Background}

The Stockholm Healthcare Services/Karolinska Institute study has not been registered at clinicaltrials.gov but on the EU site, EudraCT. The main objective is "to identify the biological mechanisms that mediate exenatideeffect in the brain, and to measure true treatment-effect of exenatide that is independent of the concurrent, symptomatic, dopaminergic treatment." The team plans to investigate the evolution of brain metabolism changes over time as measured by FDG-PET and to correlate these changes with clinical outcomes.

Title: Effect of Exenatide on disease progression in early Parkinson's Disease.

\section{Phase: 2}

Status: Recruiting

EudraCT ID: 2019-000732-26 
Sponsor: Stockholm Health Care Services, with support from the Parkinson Research Foundation of Sweden and Region Sweden. The lead investigator is Prof Per Svenningsson of the Karolinska Institute. The Feinstein Institute for Medical Research in the USA is collaborating on the trial.

\section{Estimated Enrolment: 60}

Estimated Completion Date: The trial is estimated to last for 40 months.

Study Design: The study is single-centre, double-blind, parallel group, randomised and placebo-controlled. The exenatide and placebo groups will receive treatment for 18 months, with the final evaluation occurring 3 months afterwards.

Outcome Measures: The primary outcome measure is FDG-PET analysis at baseline, 9 and 21 months.

Secondary outcomes are:

a. MDS-UPDRS part 3 in OFF-medication state, and accelerometer-based parameters of physical activity at baseline, 9, 18 and 21 months

b. MDS-UPDRS parts 1, 2, 3, and 4 in the ON-medication state, Hoehn and Yahr stage, levodopa equivalent daily dose, CSF and plasma parameters at baseline, 3, 6, 9, 12, 15, 18, and 21 months

c. PDQ-39, NMSQuest, ESS, MADRS at baseline, 6, 12, 19 months

d. MoCA at baseline, 9, and 21 months

e. B-SIT at baseline, 9 , and 18 months

\section{Comments}

The target patient population for this study is those PwP in the early stages of PD, as defined by a Hoehn and Yahr score of $\leq 2$ in the ON state, currently on levodopa therapy. A 3-month washout period at the end of the treatment phase is intended to minimise any symptomatic relief potentially provided by exenatide.

This study will provide analysis of changes in brain metabolism that will provide further insight to the mechanism of action of GLP-1 agonists in PD progression. In addition, it will produce further data on the clinical effect of exenatide in PD.

\section{Peptron Inc - PT320}

\section{Background}

Peptron Inc of South Korea has developed SmartDepot ${ }^{\mathrm{TM}}$, an ultrasonic spray drying technology that produces sustained release (SR) injectable microspheres of peptide drugs. In the case of exenatide, the gradual release of active drug will reduce the dosage frequency with the objective of sub-cutaneous (sc) injection every two weeks. The product is codenamed PT320. It claims to have higher blood-brain barrier penetration and better patient compliance.

Title: SR-Exenatide (PT320) to Evaluate Efficacy and Safety in Patients With Early Parkinson's Disease

Phase: $2 \mathrm{a}$

Status: Recruiting.

Clinicaltrials.gov ID: NCT04269642

Sponsor: Peptron Inc.

Estimated Enrolment: 99 
Estimated Completion Date: December 2021.

Study Design: This is a multi-centre (3 locations) randomised, double-blind, placebo-controlled, parallel group study. There are 3 arms - placebo; PT320 2.0mg once weekly; and PT320 2.5mg every 2 weeks with placebo administered every other week.

Outcome Measures: The primary outcome measure is motor function as measured by MDS-UPDRS part 3 at baseline and 48 weeks.

Secondary outcome measures are:

1. Specific to non-specific binding ratio (SNBR) by PET scan at baseline and 48 weeks.

2. MDS-UPDRS part 3 score at 0,24 and 60 weeks.

3. MDS-UPDRS parts 1,2 and 4 at baseline, 24,48 and 60 weeks.

4. Korean PD Questionnaire-39 at 0,48 and 60 weeks.

5. Montreal cognitive assessment-Korean (MoCA-K) at baseline, 24,48 and 60 weeks.

6. Korean-non-motor symptoms scale (K-NMSS) at baseline, 24,48 and 60 weeks.

7. The percentage of subjects in each modified Hoehn and Yahr stage at baseline, 24,48 and 60 weeks.

8. Change in levodopa dosage at baseline, 2, 4, 8, 12, 24, 36, 48 and 60 weeks. This outcome measure is the starting time of levodopa treatment and the percentage of subjects taking levodopa.

The study will also measure pharmacokinetic parameters in plasma and CSF, together with anti-exenatide antibodies, at baseline, 2, 12, 24, 36, 48 and 60 weeks.

\section{Comments}

The inclusion criteria are for PwP between the ages of 40 and 75 diagnosed in the previous 2 years. They may already be on dopaminergic therapy (less than $600 \mathrm{mg}$ levodopa per day) or not yet have started. The Hoehn and Yahr stage must be $\leq 2.5$.

The Bydureon formulation of exenatide is already a sustained release product, enabling weekly dosing. The Peptron product will need to prove superiority in efficacy, or sufficiently better convenience, to be clinically competitive.

\section{University of Florida}

\section{Background}

Aforementioned studies of exenatide in PD have suggested that the medication causes symptomatic improvement as well as potential slowing of the disease progression. This single-center open-label study intends to evaluate the effect of exenatide exposure over 1-year on the structure and function of the brain, assessed with specific MRI brain metrics.

Title: Effects of Exenatide on Motor Function and the Brain

\section{Phase: 1}

Objective: The purpose of this study is to investigate how the brain structurally and functionally changes over time in response to exenatide. A secondary objective is to investigate symptomatic changes over time in response to exenatide, in the motor, cognitive, and quality of life domains.

Status: Recruiting 
Sponsor: University of Florida

Collaborator: National Institute of Neurological Disorders and Stroke (NINDS)

Estimated Enrollment: 15 participants

Estimated Completion Date: April 2021

Study Design: This is a single center, open-label, single group assignment study. All participants will receive the intervention of exenatide $2 \mathrm{mg}$, administered as a weekly subcutaneous injection for the 1-year duration of the study.

Eligible participants are aged 40 to 77 with a diagnosis of PD within 5 years of screening and Hoehn and Yahr stage less than or equal to 2. Main exclusion criteria include individuals with previous exposure to exenatide, diagnosis of Diabetes, renal failure, "psychiatric disorders" or dementia defined as MoCA $<23$. Because of the MRI imaging obtained during the study, individuals with any implantable electrical device or metallic hardware, or with significant claustrophobia, are excluded.

\section{Outcome Measures}

This study's Primary Outcome Measures are changes in four functional and structural MRI brain imaging metrics from baseline to the study end at 1 year. The four metrics being measured include: 1) free-water accumulation in the substantia nigra, 2) blood oxygen level-dependent (BOLD) signal in the posterior putamen, 3) BOLD signal in the primary motor cortex (M1), 4) BOLD signal in the supplemental motor area (SMA).

Additional measures include questionnaires regarding quality of life and depression, motor and strength function tests and cognitive tests.

Comments: This study is unique in that it is a single-center open-label study of exenatide with only one treatment arm and no placebo arm. The lack of a placebo group for comparison of MRI metric changes from baseline to 1-year may limit the study's ability to formulate conclusions of exenatide's effect on brain structure and function, though it is unclear if the study team plans to compare the treatment group to preexisting control MRIs. The small sample size also has the potential to be a constraint in finding significance of the study's outcomes. The fact that this study's primary outcome is change in MRI imaging measures sets it apart from concurrent GLP-1 agonist trials.

\section{References}

[1] https://www.centerwatch.com/clinical-trials/listings/197110/parkinsons-disease-effects-exenatide-onmotor/

\section{NLY01 - Neuraly}

\section{Background}

NLY01 is a novel exenatide based compound developed by Neuraly Inc, a Johns Hopkins University start up biotech company. The NLY01 compound is a pegylated form of exendin-4 (exenatide), which binds to glucagonlike peptide-1 receptors (GLP-1R), which are expressed on glial brain cells. Pegylation of compounds has been shown to increase their permeability through the otherwise difficult-to-penetrate blood-brain-barrier (BBB). In keeping with this expected property, NLY01 has been shown to cross the BBB in mice. This increases compound access to the target central GLP-1R receptors, which in theory potentiate its ability to exert a therapeutic effect. 
Additionally, pegylation prolongs a molecule's half-life, so NLY01 holds out the prospect of the longest halflife of all GLP-1 agonists. Studies of NLY01 in two mouse models of PD showed that in both models it limited neuronal death, decreased formation of an inflammatory cascade and neurotoxic astrocytes, and limited motor function decline. Additionally, one mouse model had an increase in survival following NLY01 treatment. Given this pre-clinical evidence, NLY01 is being developed as a potential disease-modifying agent for PD.

Title: Multicenter, Randomized, Double-blind, Placebo-controlled Study to Evaluate the Efficacy, Safety, and Tolerability of 36 Weeks of Treatment With NLY01 in Early-stage Parkinson's disease.

Phase: 2

Objective: To assess the safety, tolerability and efficacy of NLY01 in subjects with early and untreated PD. To collect data on the effect of the drug on striatal dopaminergic binding (DaT).

Status: Recruiting

Clinicaltrials.gov ID: NCT04154072

Sponsor: Neuraly, Inc.

Estimated Enrolment: 240 participants

Estimated Completion Date: April 2021

Study Design: This is a Phase 2 randomized, placebo-controlled double-blind study comparing three parallel assigned arms: NLY01 2.5mg injection, NLY01 5mg injection, and placebo saline injection. Subjects will be randomized 1:1:1 to these three arms. The drug or placebo is delivered via weekly subcutaneous injection.

The study includes individuals aged 30 to 80 with a diagnosis of Parkinson's disease within 5 years prior to screening. Additionally, participants complete a DaTScan which must be consistent with a PD diagnosis. Notably, individuals who have used dopaminergic treatment or MAO-B inhibitors for more than 28 days, or who have had previous surgical treatment, are excluded from participation in the study. Those found to have major depression or suicidal ideation within 1 year of screening will be excluded.

\section{Outcome Measures:}

In addition to the primary outcomes of safety and tolerability, the study is assessing efficacy as measured by change from baseline to 36 weeks in the Unified Parkinson's Disease Rating Scale (MDS-UPDRS) Parts II and III combined score, for each dose of NYL01 compared to placebo. MDS-UPDRS Part II assesses motor aspects of daily living, and Part III assesses motor signs of Parkinson's.

Secondary efficacy endpoints include change from baseline in:

- UPDRS Part I: Non-Motor Aspects of Experiences of Daily Living

- Clinical Global Impression Scale-Severity (CGI-S)

- Patient Global Impression of Severity (PGI-S)

- Schwab and England Activities of Daily Living Scale (SE-ADL)

- Parkinson's Disease Questionnaire-39 (PDQ-39)

- Montreal Cognitive Assessment (MoCA)

- Scales for Outcomes of Parkinson's Disease Cognition (SCOPA-COG)

- Non-Motor Symptoms Scale (NMSS) 
Other Secondary Assessments include change from baseline in:

- DaTscan (quantitative)

- Incidence of anti-NLY01 antibodies

- NLY01 population PK assessment

Safety and Tolerability Assessments include:

- Incidence, severity, and duration of all reported treatment-emergent adverse events (TEAEs)

- Vital signs, physical examinations, and ECGs

- Clinical laboratory assessments

- Columbia-Suicide Severity Rating Scale (C-SSRS)

- Epworth Sleepiness Scale (ESS)

- Questionnaire for Impulsive-Compulsive Disorders in Parkinson's Disease-Rating Scale (QUIP-RS)

- Beck Depression Inventory-II (BDI-II)

\section{Comments}

The unique quality of NYL01 as a pegylated version of the previously-studied exenatide confers a potential advantage over the more well-known compound. By increasing blood-brain-barrier penetration with pegylation, NYL01 may have increased target engagement of central GLP-1 receptors, which in turn could prove more efficacious than its un-pegylated counterpart. This would be an additional benefit to the anticipated welltolerated safety profile, given the robust safety data for existing GLP-1 agonists.

\section{References}

[1] https://parkinsonsnewstoday.com/2018/07/20/neuraly-raises-36-million-to-advance-nly01-potentialparkinsons-therapy-into-clinical-trial/

[2] http://www.neuralymed.com/index.php/development/nly01/

\section{Liraglutide - Cedars-Sinai Medical Center}

\section{Background}

Liraglutide, a new GLP-1 mimetic with a longer half-life than exenatide, is approved by the FDA to treat adults with Type 2 Diabetes and obesity, but it is an investigational drug for possible PD treatment. Liu W et al. investigated the neuroprotective effects of liraglutide in the 1-methyl-4-phenyl-1,2,3,6-tetrahydropyridine (MPTP) mouse model of PD. Once daily injections of liraglutide $(25 \mathrm{nmol} / \mathrm{kg})$ showed prevention of MPTPinduced motor impairment, reduced apoptotic signaling, and increased anti-apoptotic signaling.

Title: A Phase II, Randomized, Double-blinded, Placebo-controlled Trial of Liraglutide in Parkinson's Disease

Phase: 2

Objective: This study's purpose is to test the efficacy and safety of liraglutide in the treatment of patients with Parkinson's disease.

Status: Recruiting

Clinicaltrials.gov ID: NCT02953665

Sponsor: Cedars-Sinai Medical Center

Collaborators: The Cure Parkinson's Trust, Novo Nordisk A/S 
Estimated Enrolment: 57 participants

Estimated Completion Date: December 2020

\section{Study Design}

This is a single center, double-blind, randomized placebo-controlled study. Subjects are randomized 2:1 to receive once daily self-administered injections of liraglutide (1.2 or $1.8 \mathrm{mg}$, as tolerated after a 2-week titration) or placebo at the same dose range. Participants will be followed for up to 56 weeks and will complete 9 inperson visits and 2 telephone visits.

Eligible participants are those aged 25 to 85 with a diagnosis of idiopathic PD for at least 2 years. Notable exclusionary criteria include individuals with dementia (Mattis Dementia Rating Scale, MADRS-2, score $<120$ ), severe depression (BDI score $>29$ ), Diabetes diagnosis, prior intracerebral surgical intervention, or current treatment with an anti-cholinergic medication.

\section{Outcome Measures}

Primary Outcome Measures are: 1) change in motor function, determined by the change in MDS-UPDRS Part III scores at baseline, 28 and 54 weeks, 2) change in non-motor function determined by the change in Non-Motor Symptoms Scale (NMSS) score at baseline, 28 and 54 weeks, and 3) change in cognitive function, determined by the change in the MADRS- 2 score at baseline, 28 and 54 weeks.

Secondary Outcome Measures include: 1) change in peripheral insulin resistance, measured by the change in Homeostasis Model Assessment (HoMA-IR) index at baseline, 28 and 54 week visit, 2) change in the total MDS-UPDRS scores at baseline, 28 and 54 week visit, 3) change in quality of life determined by change in PDQ-39 scores at baseline, 28 and 54 weeks, 4) adverse events frequency, which will be monitored with vital signs, ECGs, urine and blood tests, at screening and follow-up visits.

Comments: Liraglutide has the advantage of being a re-purposed drug with established regulatory approval for Diabetes and obesity, and thus has an established safety and tolerability profile. Also similar between these two compounds is the demonstration of their neuroprotective effects in mouse models of PD, which proved superior to exenatide's effects in these preclinical studies. A disadvantage of the drug is its once-daily injection, which will likely be less favorable to potential participants compared to exenatide and other once-weekly injected GLP-1 agonists under study (NLY01 and semaglutide).

\section{References}

[1] Liu W et al. (2015) Neuroprotective effects of lixisenatide and liraglutide in the 1-methyl-4-phenyl1,2,3,6-tetrahydropyridine mouse model of Parkinson's disease. Neuroscience. 303, 42-50.

[2] https://clinicaltrials.gov/ct2/show/NCT04269642

\section{Oslo University Hospital - Semaglutide}

\section{Background}

The primary indication for GLP-1 agonists is in the treatment of type 2 diabetes mellitus (T2DM). There have been intensive efforts in the research and development of newer, more effective and convenient drugs.

The most recent molecule to be launched is semaglutide, a weekly injectable dosage form branded as Ozempic by Novo Nordisk and approved by the FDA in 2017. An oral form of semaglutide, to be called Rybelsus, was approved by the FDA towards the end of 2019 and by the EMA in January 2020. The discovery and development of liraglutide and semaglutide has been well reviewed by Knudsen and Lau [1]. 
Ozempic claims superior glycemic control, as measured by the reduction in haemoglobin A1c, and greater weight loss than Trulicity (dulaglutide) and Bydureon (exenatide) [2]. The Oslo University Hospital team now plan to evaluate a once-weekly injection of semaglutide against placebo in PD.

Title: GLP1R in Parkinson's Disease (GIPD)

Phase: 2

Status: The official status on clinicaltrials.gov is "not yet recruiting" although the last update was in 2018.

Clinicaltrials.gov ID: NCT03659682

Sponsor: Oslo University Hospital.

Estimated Enrolment: 120

Estimated Completion Date: December 2024.

Study Design: The study is a double-blind, placebo-controlled, randomised 1:1, crossover, delayed start design, run in a single centre. Treatment in the first stage lasts for 2 years. This is followed by an open second stage where all participants will be on semaglutide, with treatment lasting for a further 2 years.

Outcome Measures: The primary outcome measure is motor function as measured by MDS-UPDRS part 3 in the OFF state at baseline and 48 months.

There are no official secondary outcomes specified, although the text describes further parameters that will be assessed:

a. Motor function measured by levodopa equivalent dose (LED).

b. Nigrostriatal degeneration measured by changes in DATscan.

c. Cognitive function measured by MME and MOCA.

d. Quality of life measured by EQFDQ and PDQ.

e. Non-motor symptoms measured by NMSS.

f. Inflammatory markers measured in blood and CSF.

Each of the above will be measured at baseline, 12, 24, 36 and 48 months. In addition, the blood and CSF samples will be used to assess the permeability of the blood brain barrier to semaglutide.

Comments: The target population for this trial is patients newly diagnosed with PD, confirmed by DaTscan. Enrolment must happen within a year of diagnosis. The eligible age range is $40-75$, narrower than many other PD trials. Ozempic is available in 2 doses, $0.5 \mathrm{mg}$ and $1.0 \mathrm{mg}$; the Oslo PD study will test the higher dose.

Given the comparatively slow degeneration rate in $\mathrm{PD}$, the treatment periods of 2 years each are a positive feature of this study. Assuming no other confounding factors, the first 2-year block should reach enough decline in the placebo group to enable a meaningful comparison with the active drug.

Semaglutide is the first GLP-1 agonist to be taken orally. This could be a strong competitive edge if the efficacy of GLP-1 agonists in PD should prove to be a class effect with similar outcomes.

\section{References}

[1] Knudsen L, Lau J (2019) The discovery and development of liraglutide and semaglutide. Front Endocrinol (Lausanne) 10, 155.

[2] Ozempic website https://www.ozempicpro.com/about-ozempic/ozempic-overview.html 


\section{Lixisenatide - University Hospital Toulouse}

\section{Background}

Lixisenatide is a GLP-1R agonist FDA approved for Type 2 Diabetes (brand name Adlyxin). It is a welltolerated daily subcutaneous injection. Lixisenatide has measurable drug concentration in the brain, and the potential neuroprotective benefits of this compound have been studied in several models. In rodent models of Alzheimer's, the compound increased neurogenesis and decreased microglial activation. In several in-vitro models of neurodegeneration, Lixisenatide showed higher efficacy of GLP-1R activation and more effective neuroprotection than exenatide and liraglutide. In the same aforementioned study of liraglutide in the MPTP mouse model of $\mathrm{PD}$, Liu $\mathrm{W}$ et al found that lixisenatide $(10 \mathrm{nmol} / \mathrm{kg})$ prevented motor impairment, reduced pro-apoptotic signaling, and increased anti-apoptotic signaling. In this same study, exenatide did not show any of these neuroprotective effects at the chosen dose $(10 \mathrm{nmol} / \mathrm{kg})$.

Title: Multicenter, Randomized, Placebo-controlled, Double Blinded, Parallel Arm Proof-of-concept Trial of Lixisenatide in Patients With Early Parkinson's Disease

\section{Phase: 2}

Objective: The study's main objective is to evaluate the effect of lixisenatide ( $20 \mu \mathrm{g} / \mathrm{d})$ versus placebo, as addon therapy to usual antiparkinsonian treatment, on the progression of motor disability in early PD patients. The goal is to assess the drug's potential "disease-modifying" effect.

Status: Recruiting

Clinicaltrials.gov ID: NCT03439943

Sponsor: University Hospital, Toulouse

Collaborators: Sanofi, Cure Parkinson's Trust, Réseau NS-Park, EUCLID

Estimated Enrolment: 158 participants

Estimated Completion Date: December 2021

Study Design: This is a multi-center randomized double-blind placebo-controlled proof-of-concept Phase 2 trial. It is a 2-arm study, with participants randomized 1:1 to either lixisenatide or placebo. The Lixisenatide group will receive lixisenatide $10 \mu \mathrm{g}$ /day for 14 days and then $20 \mu \mathrm{g}$ /day, administered via once-daily subcutaneous injections for 12 months. Participants unable to tolerate the $20 \mu \mathrm{g}$ /day dose can have their dose reduced to $10 \mu \mathrm{g} /$ day. After 12 months of treatment period there will be a 2-month wash-out period.

The study includes individuals aged 40 to 75 years who have early-stage PD, defined as PD diagnosis for less than 3 years without dyskinesias or motor fluctuations. Participants must have an optimized and stable PD medication regimen, including dopamine agonist, levodopa, and/or MAO B-inhibitor) for one month prior to the baseline study assessment. Participants are expected to remain on their medication regimen for at least the first 6 months, and ideally the full 12 months, of the study. Notably, individuals will be excluded from participation if they have had previous GLP-1 agonist exposure, have participated in another interventional trial within 30 days, or have had surgical intervention for Parkinson's. Medical conditions that prevent participation include major depression, cognitive impairment (MoCA score <26), Type 1 or 2 Diabetes, have had a weight change more than $5 \mathrm{~kg}$ in 3 months prior to screening, hyperthyroidism or uncontrolled hypothyroidism.

Outcome Measures: The Primary Outcome Measure is the change from baseline to end-point (month 12) in the MDS-UPDRS Part III motor sub-score, evaluated in the "ON" state. 
Comments: A distinct advantage of lixisenatide is that it is a repurposed drug, rather than a novel compound. As it is FDA-approved for Diabetes, it has a well-established safety and tolerability in humans. The aforementioned preclinical work in neurodegenerative and Parkinson's models suggests that lixisenatide may be more effective than exenatide at engaging the GLP-1R target, reducing inflammation and promoting neuroprotection. An advantage of this study design for participants is that they do not have to be treatment naive; they can be on a stable Parkinson's medication regimen during study participation. A disadvantage for participants is that the drug is administered as daily injections, as compared to weekly injections for other compounds (exenatide, NLY01 and semaglutide).

\section{References}

[1] Hunter, Kerry; Hölscher, Christian. Drugs developed to treat diabetes, liraglutide and lixisenatide, cross the blood brain barrier and enhance neurogenesis. BMC Neuroscience. March 2020; 13 (1): 33

[2] Liu W et al. Neuroprotective effects of lixisenatide and liraglutide in the 1-methyl-4-phenyl-1,2,3,6tetrahydropyridine mouse model of Parkinson's disease. Neuroscience. Sept 2015; 303: 42-50 